\title{
The Effect of Hypothermic and Cryogenic Preservation on Engineered Neural Tissue
}

\author{
Adam G.E. Day, BEng, PhD,, ${ }^{1, *}$ Kulraj Singh Bhangra, MEng, MRes, ${ }^{1, *}$ Celia Murray-Dunning, BSc, PhD, \\ Lara Stevanato, BSc, $\mathrm{PhD}^{2}$, and James B. Phillips, BSc, $\mathrm{PhD}^{1}$
}

This study explored different approaches to preserve engineered neural tissue (EngNT), a stabilized, cellular collagen hydrogel containing columns of aligned Schwann cells for nervous system repair. The ability to preserve EngNT without disrupting cellular and extracellular components and structures is important for clinical translation and commercialization. Stabilized cellular gels and EngNT constructs were preserved under various conditions and cell survival assessed using live/dead microscopy and metabolic assay. Optimal survival was recorded in hypothermic $\left(4^{\circ} \mathrm{C}\right)$ conditions for $2-$ 3 days using Hibernate ${ }^{\circledR}$-A media and, for longer-term cryogenic storage (liquid nitrogen), using a mixture of $60 \%$ Dulbecco's modified Eagle's medium, 30\% fetal bovine serum, and 10\% dimethyl sulfoxide. Functionality and structure of preserved EngNT were assessed in coculture with dorsal root ganglion neurons, which indicated that alignment of Schwann cells and the ability of EngNT to support and guide neuronal regeneration were not disrupted. The identification of conditions that preserve EngNT will inform development of storage and transport methodologies to support clinical and commercial translation of this technology and other therapies based on cellular hydrogels.

Keywords: collagen, Schwann cell, preservation, hypothermic, cryogenic, nerve guide, nerve regeneration, nerve tissue engineering, hydrogel

\section{Introduction}

$\mathbf{E}$ NGINEERED NEURAL TISSUE (EngNT) is an aligned cellular biomaterial that can be formed from a combination of Schwann cell self-alignment and stabilization in collagen gels. ${ }^{1}$ Our previous work showed EngNT to be a potential alternative to the current clinical gold standard autograft for treating peripheral nerve injuries, with the ability to support neuronal regeneration and the capacity to be constructed using therapeutic cells. ${ }^{1-4}$ To support commercialization and clinical translation of EngNT, it would be beneficial to be able to store the living cellular constructs in such a manner that preserves their cell viability and architecture. This would allow flexibility in the timing between manufacture and usage, enabling manufacturing to be conducted in a specialist facility with subsequent distribution to clinical centers. Furthermore, the ability to store an allogeneic off-the-shelf formulation of EngNT could allow clinics to maintain stock locally.
The two main methods used to preserve cells and tissues are hypothermic and cryogenic storage. Successful hypothermic preservation of tissue, which involves refrigeration rather than freezing of samples, has been shown using Collins solution and University of Wisconsin solution (marketed as ViaSpan). ${ }^{5-7}$ Effective hypothermic preservation of neuronal cells has been shown using Hibernate ${ }^{\circledR}-A$, Hibernate-E, and HypoThermosol $^{\circledR}$ FRS (HTS-FRS). ${ }^{8-10}$ Brewer et al. have shown that Hibernate-E (Hibernate-E has a higher osmolality than Hibernate-A; 240 and $280 \mathrm{mOsm} / \mathrm{kg} \mathrm{H}_{2} \mathrm{O}$, respectively) can successfully preserve brain tissue, ${ }^{11}$ although there is no literature exploring the ability of these solutions to preserve nerve grafts or EngNT. The most common way to preserve cells/tissue cryogenically is with the use of the cryoprotectant dimethyl sulfoxide (DMSO). ${ }^{12,13}$ Kawamoto et al. demonstrated that freezing neurons with 5-10\% DMSO was optimal for survival ${ }^{14} ;<5 \%$ DMSO did not provide enough protection to cells from freezing effects and above $10 \%$ DMSO was toxic to cells. Similar to cryopreserving cell suspensions, $10 \%$

\footnotetext{
${ }^{1}$ Department of Biomaterials and Tissue Engineering, UCL Eastman Dental Institute, University College London, London, United Kingdom.

${ }^{2}$ ReNeuron, Pencoed, Bridgend, Wales.

*These authors contributed equally to this work.

(c) Adam G.E. Day et al. 2017; Published by Mary Ann Liebert, Inc. This is an Open Access article distributed under the terms of the Creative Commons Attribution License, which permits unrestricted use, distribution, and reproduction in any medium, provided the original work is properly cited.
} 
DMSO has also been found to be optimal when cryopreserving primary nerve tissue. ${ }^{13}$ For example, Das et al. ${ }^{15}$ showed that cryopreserving rat neocortical and brainstem tissues using 2-4\% DMSO was inferior to using $10 \%$ when subsequently transplanted into the cerebellum of neonatal host rats. Hashimoto et al. ${ }^{16}$ showed that cryopreserving bovine brain tissue with $4 \%$ or $8 \%$ DMSO was inferior to $10 \%$. Concentrations of DMSO higher than 10-15\% have also been found to reduce cell survival when cryopreserving rat, ${ }^{17}$ bovine, ${ }^{16}$ and human tissues. ${ }^{18}$ Fansa et al. reported harvesting nerve grafts from rats and storing them cryogenically using Dulbecco's modified Eagle's medium (DMEM) and 10\% DMSO. ${ }^{19}$ After $24 \mathrm{~h}$, nerve grafts were transplanted back into the same animal and left for 6 weeks. Some nerve graft samples were histologically analyzed without reimplantation to determine whether freezing altered the structural features of the grafts. Although structural features of the grafts stored with a cryoprotectant were not altered, morphometric results after 6 weeks showed that cryopreserved grafts were inferior at supporting regeneration as fewer axons and less myelination were found compared with samples that were not exposed to any cryogenic conditions. The authors speculate that impaired regeneration could be attributed to delayed Wallerian degeneration and slower revascularization. Furthermore, a reduced survival of resident Schwann cells in the cryogenically stored graft may have impaired regeneration. ${ }^{19}$

The aim of this study was to investigate the effects of a range of hypothermic and cryogenic storage approaches on the viability, structure, and function of EngNT seeded with rat Schwann cells. The key outcome measures under consideration were the viability of the cells within the constructs and preservation of the architecture of EngNT. Storage media and protocols were selected and compared using simplified tissue constructs to investigate cell viability after different storage periods, then the ability of promising candidate conditions to preserve EngNT structure and function was assessed.

\section{Materials and Methods}

\section{Production of cellular collagen gels}

Collagen hydrogels containing Schwann cells were manufactured according to previously established protocols and stabilized using plastic compression. ${ }^{20}$ Schwann cells (SCL 4.1/F7; Health Protection Agency, United Kingdom) were maintained in DMEM $\left(\right.$ Gibco $\left.^{\circledR}\right)$ supplemented with penicillin and streptomycin $(100 \mathrm{U} / \mathrm{mL}$ and $100 \mathrm{mg} / \mathrm{mL}$, respectively; Sigma, United Kingdom) and $10 \% \mathrm{v} / \mathrm{v}$ fetal bovine serum (FBS; Gibco) in standard cell culture flasks in a $37^{\circ} \mathrm{C}$ incubator with $5 \%$ carbon dioxide $\left(\mathrm{CO}_{2}\right)$ and passaged with trypsin/EDTA (Life Technologies, United Kingdom) when required. Acid-solubilized type I bovine collagen $(3 \mathrm{mg} / \mathrm{mL}$; Koken, Japan) was diluted with $26.8 \%$ v/v $1 \mathrm{mM}$ hydrochloric acid to a concentration of $2 \mathrm{mg} / \mathrm{mL}$. This solution was then mixed with $10 \% \mathrm{v} / \mathrm{v} 10 \times$ minimum essential medium (Sigma) and neutralized $(\sim 0.5 \% \mathrm{v} / \mathrm{v}$ neutralizing solution; Lonza Bioscience, United Kingdom) before addition of $10 \% \mathrm{v} / \mathrm{v}$ culture media containing Schwann cells. To produce simple unaligned gels for initial experiments, $1 \mathrm{~mL}$ of cellular collagen solution $\left(2 \times 10^{6}\right.$ cells $\left./ \mathrm{mL}\right)$ was cast per well of a 24 -well plate. To manufacture aligned gels for EngNT production, $400 \mu \mathrm{L}$ of cellular collagen solution $\left(4 \times 10^{6}\right.$ cells $\left./ \mathrm{mL}\right)$ was added to tethering molds that allowed self-alignment. ${ }^{21} \mathrm{Un}$ - aligned cellular collagen gels were used for the initial experiments because a higher throughput could be achieved and a greater number of identical pieces could be obtained from simple unaligned gels compared with EngNT. Gels were incubated at $37^{\circ} \mathrm{C}$ for $15 \mathrm{~min}$ to set. Unaligned collagen gels were stabilized for 15 min using the RAFT ${ }^{\mathrm{TM}}$ absorber (Lonza Bioscience). Tethered gels were immersed in culture media and incubated for $24 \mathrm{~h}$ to allow cellular self-alignment, ${ }^{1}$ then media removed before stabilization using RAFT to generate EngNT.

\section{Preservation conditions}

Unaligned cellular collagen gels were used in initial experiments to determine the appropriate preservation conditions for cell survival. Each circular gel was cut radially into six equal sectors that were transferred to six different preservation conditions for 2 days. Three pieces were preserved in hypothermic conditions (refrigerated at $4^{\circ} \mathrm{C}$ ): (1) Hibernate-A (Gibco), (2) DMEM 10\% FBS, and (3) HTS-FRS (BioLife Solutions). Three pieces were preserved in cryogenic conditions through controlled rate cooling using (4) 80\% DMEM $+10 \% \mathrm{FBS}+10 \%$ DMSO, (5) $60 \%$ DMEM $+30 \% \mathrm{FBS}+10 \%$ DMSO, and (6) $90 \%$ HTS-FRS $+10 \%$ DMSO (Mr. Frosty ${ }^{\mathrm{TM}}$ freezing container for 1 day in a $-80^{\circ} \mathrm{C}$ freezer, followed by 2 days in liquid nitrogen). Fresh gels not exposed to preservation conditions were used as controls. These were analyzed immediately by live/dead assay using Syto-9 (5 $\mu \mathrm{M}$; Life Technologies) and propidium iodide $(20 \mu \mathrm{g} / \mathrm{mL}$; SigmaAldrich, United Kingdom), respectively, diluted in cell culture media, and incubated at room temperature for $1 \mathrm{~min}$ before viewing. Preserved samples were rapidly warmed in a $37^{\circ} \mathrm{C}$ water bath after the preservation period and then live/dead assay performed as described above. Further experiments were conducted on unaligned gels and EngNT using the following preservation conditions: Hibernate-A, HTS-FRS, 60\% DMEM $+30 \%$ FBS $+10 \%$ DMSO, and $90 \%$ HTS-FRS $+10 \%$ DMSO (unaligned gels only) using the methods described above for durations of 2, (EngNT only), 7, and 14 days at $4^{\circ} \mathrm{C}$ or in liquid nitrogen. Samples stored in liquid nitrogen were first cooled to $-80^{\circ} \mathrm{C}$ (Mr. Frosty freezing container for 1 day). For testing aligned cellular gels, EngNT constructs were cut into quarters, unless otherwise stated.

\section{Metabolic assessment of EngNT}

CellTiter-Glo $^{\circledR}$ (Promega, United Kingdom) was used to quantify the presence of viable Schwann cells in EngNT before and after preservation in Hibernate-A for 3 days. Each EngNT sample was cut transversely into two equal pieces, each containing $0.8 \times 10^{6}$ cells, one of which was tested immediately (baseline control) and the other subjected to preservation for 3 days at $4^{\circ} \mathrm{C}$ in Hibernate-A or DMEM 10\% FBS, then returned to standard culture conditions $\left(37^{\circ} \mathrm{C} 5 \% \mathrm{CO}_{2}\right.$ in DMEM $10 \%$ FBS) for 1 or 3 days, then tested. Equivalent control samples were maintained under standard culture conditions for the entire duration ( 4 or 6 days). One hundred microliters of CellTiter-Glo and $100 \mu \mathrm{L}$ of DMEM $10 \%$ FBS along with each sample were added to each well of an opaque 96-well plate and placed on a shaker at $100 \mathrm{rpm}$. After $10 \mathrm{~min}$, the plate was incubated statically for a further $20 \mathrm{~min}$ at room temperature $\left(21^{\circ} \mathrm{C}\right)$. Luminescence $(\sim 528 \mathrm{~nm})$ of each sample was then read using an FLX800 $\left(\right.$ BioTek $\left.^{\circledR}\right)$ plate reader. Luminescence 
from blank control wells was subtracted from samples containing gels.

\section{Coculture of preserved EngNT with neurons}

Coculture experiments were used to investigate whether EngNT preserved in Hibernate-A or 60\% DMEM, 30\% FBS, and 10\% DMSO after 3 days would guide and support dorsal root ganglion (DRG) neurons compared with EngNT left in normal culture conditions for 3 days (no preservation). All experimental procedures involving animals were conducted in accordance with the UK Animals (Scientific Procedures) Act (1986) and approved by the relevant UCL ethics review board. Dissociated DRG neurons were prepared from a single adult (200-300 g) Wistar rat. DRGs were incubated in collagenase IV $\left(0.125 \%\right.$; Sigma) for $90 \mathrm{~min}$ at $37^{\circ} \mathrm{C}$, then dissociated by trituration and washed twice with $20 \mathrm{~mL}$ of culture medium before being incubated for $24 \mathrm{~h}$ with cytosine arabinoside $(0.01 \mathrm{mM})$ to deplete glia. The resulting cultures were enriched with neurons, ${ }^{1}$ which were washed twice with $20 \mathrm{~mL}$ of culture medium through centrifugation at $400 \mathrm{~g}$ for $5 \mathrm{~min}$ and resuspended into a final volume of $600 \mu \mathrm{L}$. After thawing cryogenically preserved samples in a $37^{\circ} \mathrm{C}$ water bath and then washing preserved EngNT samples with $37^{\circ} \mathrm{C}$ DMEM 10\% FBS, neurons were seeded through pipetting $50 \mu \mathrm{L}$ onto each surface of quartered EngNT sheets. Neurons were left in a $37^{\circ} \mathrm{C}$ humidified incubator with $5 \% \mathrm{CO}_{2}$ for $1.5 \mathrm{~h}$ to allow attachment, after which EngNT sheets were immersed in $1 \mathrm{~mL}$ of DMEM 10\% FBS and incubated for 3 days. Samples were then washed in phosphate-buffered saline and fixed in $4 \%$ paraformaldehyde for at least $24 \mathrm{~h}$ at $4^{\circ} \mathrm{C}$ before immunofluorescence staining was carried out as described previously for collagen gels ${ }^{22,23}$ to detect $\beta$ IIII-tubulin-positive neurons (1:400, anti-mouse; Sigma-Aldrich) and S100positive Schwann cells (1:400, anti-rabbit; Dako, United Kingdom). Secondary antibodies (DyLight 488 goat antirabbit IgG and DyLight 549 horse anti-mouse IgG; Vector Laboratories, United Kingdom) were then used (1:200) for $90 \mathrm{~min}$ at ambient temperature $\left(21^{\circ} \mathrm{C}\right)$ to detect the primary antibody.

\section{Scanning electron microscopy}

Scanning electron microscopy (SEM) was used to assess whether EngNT preserved in Hibernate-A or 60\% DMEM, $30 \%$ FBS, and 10\% DMSO for 3 days affected collagen alignment compared with EngNT maintained in normal culture conditions for 3 days (no preservation). Samples were fixed in $3 \%$ glutaraldehyde for at least $24 \mathrm{~h}$ at $4{ }^{\circ} \mathrm{C}$, then dehydrated through an increasing graded series of ethanol. Finally, samples were fully immersed in hexamethyldisilazane for $2 \mathrm{~min}$, which was then removed, and samples were left to dry. Samples were mounted onto aluminum SEM stubs using double-sided carbon sticky tabs (Agar Scientific, United Kingdom), sputter coated with gold palladium, and imaged using a Philips XL30 FEGSEM at $5 \mathrm{kV}$.

\section{Microscopy and image analysis}

Samples were placed onto microscope slides and imaged immediately after live/dead staining using a $20 \times$ objective on a Zeiss Axioskop fluorescence microscope. Five equivalent predetermined fields were assessed on each piece of unaligned gel to find the mean cell death percentage. Seven predetermined fields were assessed for each quartered EngNT sample.

Schwann cell and neurite alignment and growth were assessed using confocal microscopy (LSM710; Zeiss, Germany) by tile scanning the entire quarter of each EngNT sheet. Images were captured using a $20 \times$ lens, using z-stacks of $18 \mu \mathrm{m}$ for neurites and $25 \mu \mathrm{m}$ for Schwann cells with a step size of $2 \mu \mathrm{m}$. Alignment analysis was conducted using Volocity ${ }^{\mathrm{TM}}$ software (Perkin Elmer, Waltham, MA) running automated 3D image analysis protocols to measure the angle of Schwann cell and neurite alignment in each field. ${ }^{21}$

Statistical analysis was carried out using analysis of variance with Tukey's or Dunnett's post-tests (GraphPad Prism).

\section{Results}

\section{Survival of Schwann cells in preserved collagen gels}

An initial panel of preservation conditions was tested to determine what medium formulation and method (hypothermic or cryogenic) minimized cell death optimally after 2 days within unaligned collagen gels compared with baseline control gels (Fig. 1A). Ninety percent HTS FRS 10\% DMSO was included because of its similar formulation to the commercial product Cryostor ${ }^{\circledR}$. Preliminary data indicated that increasing the concentration of FBS in the formulation of cryogenic media was not beneficial to cell survival, with $25 \%$ DMEM, 65\% FBS, and 10\% DMSO and 90\% FBS and 10\% DMSO resulting in $38.8 \%$ and $37.5 \%$ cell death, respectively, after 2 days. Samples preserved hypothermically with Hibernate-A and HTS-FRS resulted in lower cell death than all other conditions. Hibernate-A samples were not significantly different in terms of cell death compared with control samples, but a significant difference was found using HTSFRS. Of the cryogenically preserved samples, the condition 60\% DMEM, 30\% FBS, and 10\% DMSO resulted in lower cell death than other cryogenic conditions, but was significantly different from the control samples. Following this initial study, the two hypothermic and cryogenic conditions associated with the lowest levels of cell death in each case were used in studies lasting 7 and 14 days (Fig. 1B). Cell death increased with storage time regardless of the preservation condition used. At day 7, hypothermic preservation using Hibernate-A only raised cell death compared with initial control samples by $7.2 \%$ to $26.2 \%$, whereas all other conditions tested showed an increase in cell death to $40-50 \%$. By day 14, cell death in Hibernate-A samples had increased to $45 \%$ and the other hypothermic condition (HTS-FRS) had increased to $72 \%$ cell death.

\section{Survival of Schwann cells in preserved EngNT}

Following initial experiments using simple, unaligned collagen gels, the survival of Schwann cells in EngNT constructs was measured after hypothermic preservation in Hibernate-A or cryogenic preservation in 60\% DMEM, $30 \%$ FBS, and $10 \%$ DMSO (Fig. 2). Similarly to the unaligned gels, cell death increased with time in samples preserved hypothermically, whereas cell death in cryogenic preservation conditions was $47 \%$ at 2 days, remained similar at 7 days, and only increased by $\sim 10 \%$ by day 14 . 

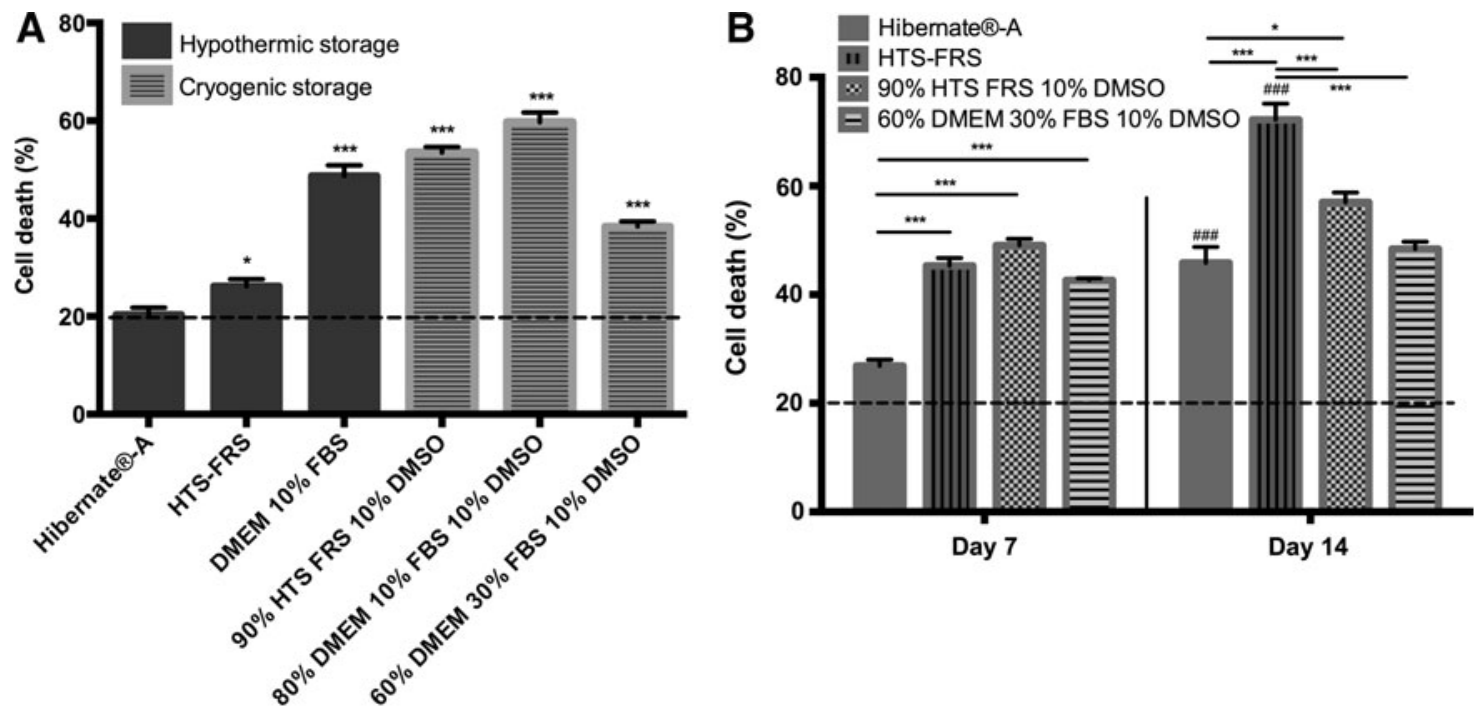

FIG. 1. Schwann cell death in unaligned gels following preservation. Unaligned gels were initially preserved in six different conditions for 2 days and compared with baseline day 0 control gels (dotted line: 19.5\% \pm 9\%) through one-way ANOVA (A). Dunnett's post-tests revealed significant differences in cell death in preserved samples compared with controls $(* p<0.05$, $* * * p<0.001)$. The two hypothermic and cryogenic conditions associated with the lowest levels of cell death in each case were then investigated further to include days 7 and 14 (B). Two-way ANOVA revealed a main effect between conditions, time, and their interaction $(p<0.001)$. Significant differences between conditions in terms of cell death at each time point were found using Tukey's post-tests $\left({ }^{*} p<0.05\right.$, $\left.* * * p<0.001\right)$. Excluding Hibernate ${ }^{\circledR}-\mathrm{A}$ at day 7 , all samples showed significantly greater cell death compared with control (day 0) samples (dotted line, 19.6\% $\pm 6.8 \%, p<0.001$ ). Samples stored hypothermically significantly increased in cell death by 14 days compared with corresponding conditions at day $7\left({ }^{\# \#} p<0.001\right)$. $n=6$, data are mean \pm SEM. ANOVA, analysis of variance; DMEM, Dulbecco's modified Eagle's medium; DMSO, dimethyl sulfoxide; FBS, fetal bovine serum; HTS-FRS, HypoThermosol FRS; SEM, standard error of mean.

Due to the substantial increase in cell death between days 2 and 7 using hypothermic storage in Hibernate-A, additional time points were investigated for this condition to explore the time course in more detail. Samples tested at days 2 and 3 were not significantly different in cell death compared with

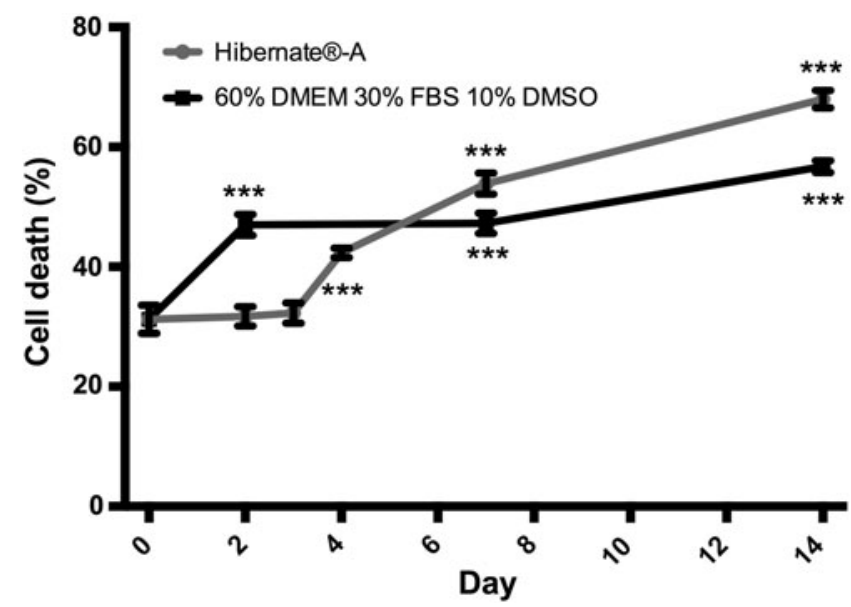

FIG. 2. Comparison of Schwann cell death (\%) in EngNT preserved using Hibernate-A (hypothermic condition) and $60 \%$ DMEM, 30\% FBS, and 10\% DMSO (cryogenic condition). After testing for 2, 7, and 14 days, further analysis of EngNT preserved using Hibernate-A was carried out to include days 3 and 4. Day 0 represents unpreserved control samples. ANOVA with Dunnett's post-tests revealed significant increases in cell death compared with controls at day $0(* * * p<0.001) . n=6$, data are mean \pm SEM. EngNT, engineered neural tissue; FBS, fetal bovine serum. control samples, then from day 4 onward, a significant increase in cell death was detected with increasing time.

\section{Metabolic activity of Schwann cells within EngNT postpreservation}

To test the viability of cells within EngNT following preservation, metabolic activity was determined after preserved samples were returned to standard culture conditions and maintained for 1 and 3 days (Fig. 3). Preserved samples and those that had been maintained in culture (DMEM 10\% FBS) for the duration had significantly decreased ATP levels compared with fresh samples analyzed immediately after construction. The samples preserved in Hibernate-A showed a significantly higher metabolic activity than equivalent samples maintained in culture at the 4-day time point, but this difference between conditions was not present after 6 days.

\section{Schwann cell and collagen alignment in EngNT and guidance of neurite growth following preservation}

Coculture experiments using primary rat DRG neurons were used to investigate whether EngNT preserved for 3 days in Hibernate-A (hypothermic) or 60\% DMEM, 30\% FBS, and 10\% DMSO (cryogenic) would guide and support DRG neurons compared with EngNT maintained under standard culture conditions (DMEM 10\% FBS) for 3 days (no preservation). Schwann cell alignment and neuronal growth and guidance did not appear to be affected by the preservation, and scanning electron micrographs indicated that collagen alignment was also unaffected by the preservation (Fig. 4A). Schwann cell alignment within preserved EngNT samples was 


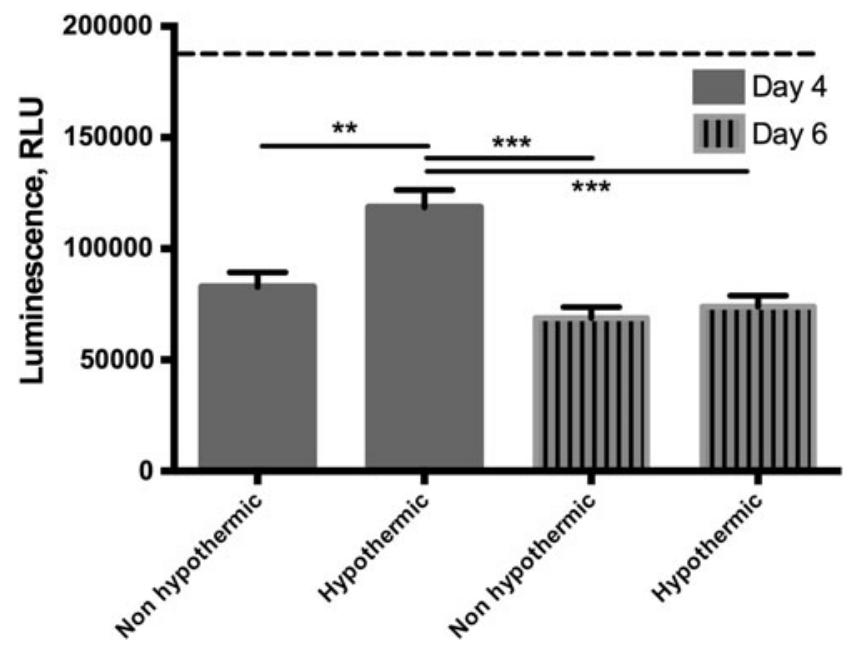

FIG. 3. Metabolic activity of EngNT with and without preservation in Hibernate-A. After 3 days in Hibernate-A, preserved samples were put back into culture conditions for 1 day (4 days total time) or 3 days (6 days total) and compared with equivalent samples maintained in culture for 4 and 6 days. Two-way ANOVA revealed a main effect between conditions $(p<0.05)$, time $(p<0.001)$, and their interaction $(p<0.05)$. Two-way ANOVA with Tukey's post-tests showed that control samples (analyzed immediately after construction) had significantly higher metabolic activity than all other samples (dotted line, 186,000 \pm 8000 ; $p<0.001)$. The hypothermic condition at day 4 had significantly higher metabolic activity than the other samples at days 4 and $6(* * p<0.01, * * * p<0.001) . n=4$, data are mean \pm SEM.

not significantly different from control samples, with $\sim 50 \%$ of cells showing a deviation of $<30^{\circ}$ from the long axis of EngNT (Fig. 4B). A similar trend was seen in the alignment of neurites with no significant difference between preserved samples, where the orientation of $61 \%$ (Hibernate-A) and 55\% (cryogenic) of neurites deviated by $<30^{\circ}$ from the long axis of EngNT, compared with 50\% in control samples (Fig. 4C).

\section{Discussion}

Our previous work showed EngNT to be a potential alternative to the current gold standard autograft and better than an empty tube for repairing critical length gaps in a rat peripheral nerve transection model. ${ }^{1,2}$ To support clinical translation of EngNT, an optimal method for storing cellular constructs between manufacture and implantation that preserves cell viability and functional architecture would be beneficial.

Experiments using unaligned cellular constructs indicated that hypothermic conditions were more appropriate for short-term storage and cryogenic conditions enabled longerterm storage, with a range of outcomes in terms of cell death using different medium preparations. The most effective cryogenic medium formulation tested here was 60\% DMEM, 30\% FBS, and 10\% DMSO, which resulted in $\sim 25 \%$ initial cell death compared with controls, but remained relatively stable over time. This 25\% initial cell death is consistent with other studies investigating storage of tissue-engineered constructs. For example, Elder et $a .^{24}$ cryopreserved collagen-based blood vessel constructs overnight using DMEM supplemented with $10 \% \mathrm{FBS}$ and $1 \mathrm{~mol} / \mathrm{L}$ DMSO and reported $25 \%$ increase in cell death compared with control samples, and Costa et $_{\text {al. }}{ }^{25}$ found decreased levels of DNA and metabolic activity from goat bone marrow stem cells cryogenically preserved in 3D porous scaffolds for 7 days using DMEM, FBS, and DMSO compared with control samples.

The most effective medium formulation tested here in terms of minimizing Schwann cell death during hypothermic storage was Hibernate-A. Short-term preservation (2 days) was better using hypothermic methods rather than cryogenic methods, which is unsurprising since at subzero conditions, ice formation in the extracellular medium creates an osmotic gradient across cell membranes that results in water being drawn out of the cells. ${ }^{13,26}$ As the samples are cooled further, water continues to be drawn out of the cells, causing cell shrinkage. This causes intracellular solutes to become concentrated that can be toxic to cells, resulting in cell death. ${ }^{13,26}$ Hibernate-A has been used to increase neuron survival during tissue dissociation and to preserve rat embryonic brain tissue ${ }^{11,27,28}$ but has never been used to preserve cells in EngNT before. In the present study, EngNT stored for up to 3 days under hypothermic conditions in Hibernate-A showed minimal increase in Schwann cell death compared with controls, whereas after storage for 4 days or longer, Schwann cell death gradually increased with time. This provides important new information about the feasibility of using hypothermic conditions to store and transport cellular biomaterials in the days immediately following manufacture.

After identification of hypothermic and cryogenic approaches that enabled simplified unaligned cellular constructs to survive, further studies were conducted to investigate whether the structure and function of EngNT were disrupted under the same conditions. After the preservation period, samples were returned to standard culture for 1 and 3 days and their metabolic activity was assessed. There was a decrease in metabolic activity in EngNT after time in culture regardless of whether preservation conditions or standard culture conditions were used. Interestingly, at day 4, the EngNT that had been stored hypothermically was more metabolically active than the equivalent sample that had been maintained at $37^{\circ} \mathrm{C}$, indicating that the baseline reduction in metabolic activity over time in vitro was reduced in hypothermically stored samples. By 6 days, there was no difference between preserved and cultured samples, which is consistent with the data obtained from the cell death studies in EngNT that also showed hypothermic preservation was most effective for up to 3 days. Kawamoto et al. ${ }^{14}$ developed an optimized preservation medium for storing fetal rat central nervous system tissue and reported a significant reduction in cell survival after 8 days, which is similar to the effect on cell survival following hypothermic storage of EngNT shown here. This medium formulation had a $\mathrm{pH}$ of 6.8-7.4 and included $30-70 \mathrm{mM} \mathrm{K}^{+}, 10-30 \mathrm{mM} \mathrm{Na}^{+}, 5-$ $50 \mathrm{mM} \mathrm{PO}^{2-}, 20 \mathrm{mM}$ lactic acid, $5 \mathrm{mM}$ glucose, and $<0.1 \mathrm{mM}$ $\mathrm{Ca}^{2+}$. This medium is similar to Hibernate-A used in this study, but contains lower concentrations of sodium and calcium. Brewer et al. ${ }^{11}$ indicated that neuron survival was higher using Hibernate-E supplemented with B27 than the media described in Ref. ${ }^{14}$ Kawamoto et al. suggested that cell death during hypothermic storage over longer time periods could be due to biophysical stresses such as ionic fluxes across cell 
A
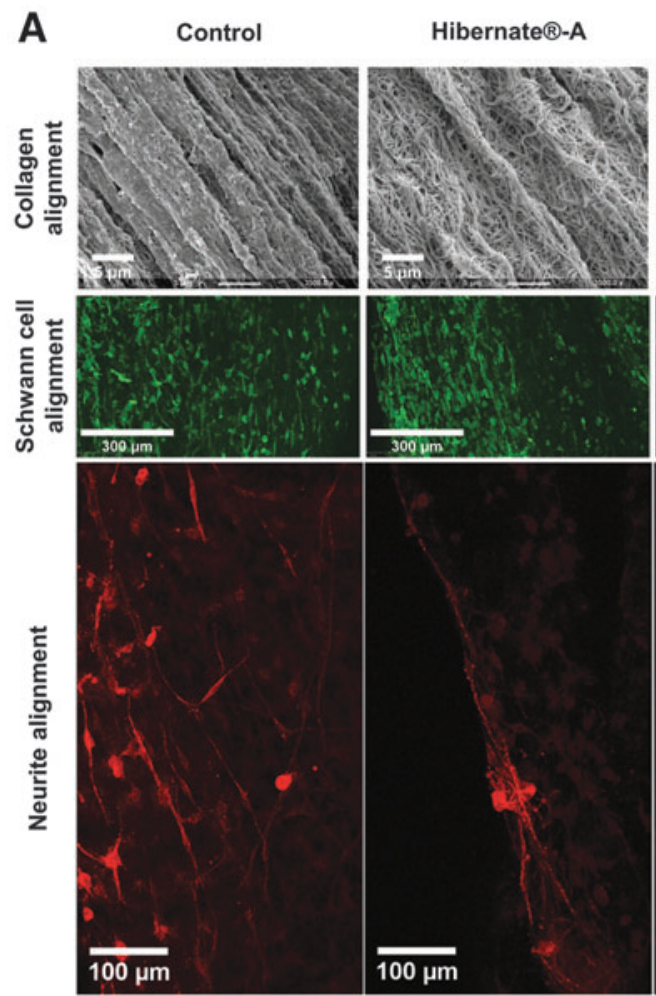
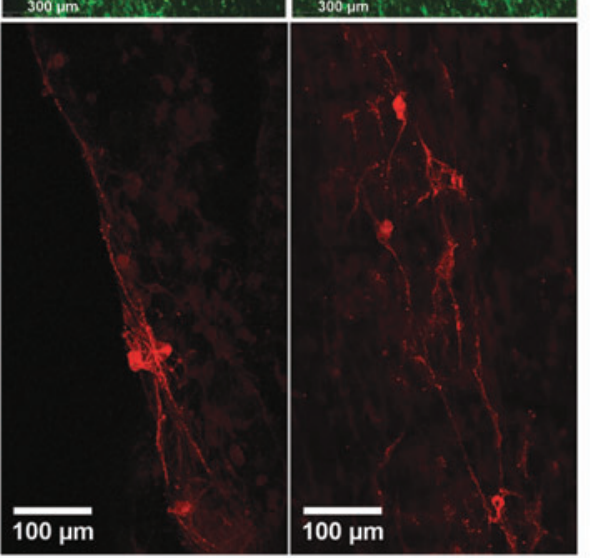

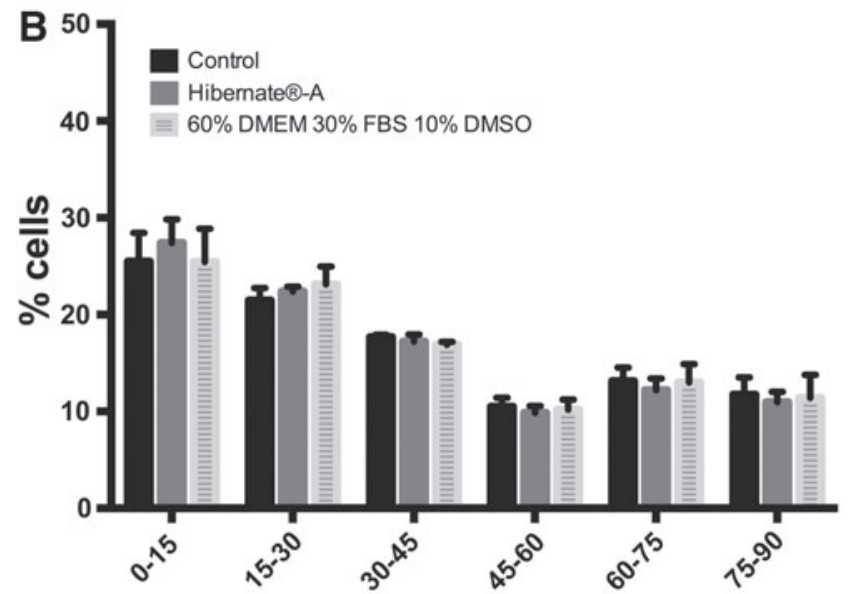

Angle of cell deviation from the long axis of the gel (degrees)

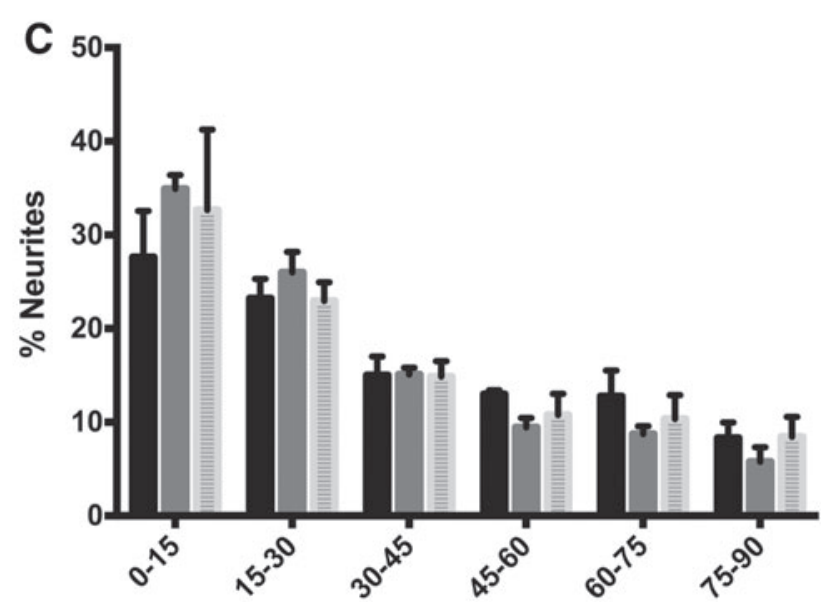

Neurite deviation from the long axis of EngNT-F7 (degrees)

FIG. 4. Collagen, Schwann cell, and neurite alignment in EngNT after cryogenic or hypothermic preservation. EngNT samples were preserved hypothermically in Hibernate-A or cryogenically in $60 \%$ DMEM, $30 \%$ FBS, and $10 \%$ DMSO or maintained in culture for 3 days, then cocultured with neurons for 3 days. Scanning electron micrographs show collagen structure, and fluorescence micrographs $(20 \times)$ show aligned Schwann cells (green, S100) and aligned neurite growth (red, $\beta$ III-tubulin) in control, hypothermic, or cryogenic EngNT samples (A). Confocal tile scan micrographs of each sample were used to calculate Schwann cell (B) and neurite $(\mathbf{C})$ alignment in relation to the long axis of EngNT $(n=4$ for preservation conditions and $n=3$ for control, data are mean \pm SEM). Color images available online at www.liebertpub.com/tec

membranes, $\mathrm{pH}$ changes due to build-up of acidic metabolites, energy deprivation, exposure to toxic debris, and degradation by proteolytic enzymes released by damaged cells. ${ }^{14}$ Subsequent studies used this hibernation approach to preserve fetal ventral mesencephalic tissue grafts successfully for a number of days under hypothermic conditions. ${ }^{29,30}$ From these studies and the results reported here, it is clear that EngNT, like natural neural tissues, can be stored for a few days under hypothermic conditions, but for longer-term storage, this will result in a reduction in cell viability, so for those situations, cryopreservation is the better option.

Alignment of Schwann cells and nanofibrillar collagen matrix in EngNT and other artificial grafts contributes to guidance of neuronal regeneration through a nerve injury site. $^{1,2,31-34}$ It was therefore important to investigate whether the anisotropic cellular and extracellular architecture in EngNT constructs was maintained following preservation in conditions that were sufficient to maintain cell viability. 
Results from confocal image analysis of Schwann cell alignment and alignment of cocultured neurons indicated that both hypothermic and cryogenic preservation methods using the optimal medium formulations and timing for cell survival were not detrimental to EngNT structure, with neurite alignment in vitro being equivalent to previous studies. ${ }^{1}$

\section{Conclusion}

Medium conditions for hypothermic and cryogenic storage of EngNT were compared and formulations were identified that supported survival of resident Schwann cells in each case. For short-term preservation of up to 3 days, Hibernate-A at hypothermic storage temperature was optimal. For longer-term preservation, cryogenic storage using a medium formulation, including 30\% FBS and 10\% DMSO, was optimal. In each case, Schwann cell alignment within EngNT was not affected and the ability of the constructs to guide neurite growth in coculture was not diminished. The results of this study will contribute to the clinical and commercial translation of EngNT and may also be of benefit to other groups seeking to preserve cellular materials for nerve repair or for other tissue engineering applications. Future work will focus on optimization of preservation of EngNT using therapeutically relevant cells under good manufacturing practice guidelines, along with testing in vivo, to improve the scale-up and clinical translation of this technology.

\section{Acknowledgment}

This project was supported by funding from Innovate-UK (Biomedical Catalyst 101599).

\section{Disclosure Statement}

No competing financial interests exist.

\section{References}

1. Georgiou, M., Bunting, S.C., Davies, H.A., Loughlin, A.J., Golding, J.P., and Phillips JB. Engineered neural tissue for peripheral nerve repair. Biomaterials 34, 7335, 2013.

2. Georgiou, M., Golding, J.P., Loughlin, A.J., Kingham, P.J., and Phillips, J.B. Engineered neural tissue with aligned, differentiated adipose-derived stem cells promotes peripheral nerve regeneration across a critical sized defect in rat sciatic nerve. Biomaterials 37, 242, 2015.

3. Martens, W., Sanen, K., Georgiou, M., Struys, T., Bronckaers, A., Ameloot, M., et al. Human dental pulp stem cells can differentiate into Schwann cells and promote and guide neurite outgrowth in an aligned tissue-engineered collagen construct in vitro. FASEB J 28, 1634, 2014.

4. Sanen, K., Martens, W., Georgiou, M., Ameloot, M., Lambrichts, I., and Phillips, J. Engineered neural tissue with Schwann cell differentiated human dental pulp stem cells: potential for peripheral nerve repair? J Tissue Eng Regen Med. 2017 [Epub ahead of print]; DOI: 10.1002/term.2249.

5. Collins, G.M., Bravo-Shugarman, M., Novom, S., and Terasaki, P.I. Kidney preservation for transplantation 1. 12-hour storage in rabbits. Transplant Proc 1, 801, 1969.

6. Toom, R.D., De Jong, M., Krenning, E.P., Hoek, H.J.V.D., Kate, F.J.W.T., Hennemann, G., et al. Euro-collins solution versus uw-solution for long-term liver preservation in the isolated rat-liver perfusion model. HPB Surg 4, 313, 1991.
7. Hendry, P.J., Labow, R.S., and Keon, W.J. A comparison of intracellular solutions for donor heart preservation. J Thorac Cardiovasc Surg 105, 667, 1993.

8. Brewer, G.J., and LeRoux, P.D. Human primary brain tumor cell growth inhibition in serum-free medium optimized for neuron survival. Brain Res 1157, 156, 2007.

9. Montoya-Gacharna, J.V., Sutachan, J.J., Chan, W.S., Sideris, A., Blanck, T.J., and Recio-Pinto, E. Preparation of adult spinal cord motor neuron cultures under serum-free conditions. Methods Mol Biol 846, 103, 2012.

10. Robinson, N.J., Picken, A., and Coopman, K. Low temperature cell pausing: an alternative short-term preservation method for use in cell therapies including stem cell applications. Biotechnol Lett 36, 201, 2014.

11. Brewer, G.J., and Price, P.J. Viable cultured neurons in ambient carbon dioxide and hibernation storage for a month. Neuroreport 7, 1509, 1996.

12. Best, B.P. Cryoprotectant toxicity: facts, issues, and questions. Rejuvenation Res 18, 422, 2015.

13. Paynter, S.J. Principles and practical issues for cryopreservation of nerve cells. Brain Res Bull 75, 1, 2008.

14. Kawamoto, J.C., and Barrett, J.N. Cryopreservation of primary neurons for tissue-culture. Brain Res 384, 84, 1986.

15. Das, G.D., Houle, J.D., Brasko, J., and Das, K.G. Freezing of neural tissues and their transplantation in the brain of rats-technical details and histological observations. J Neurosci Methods 8, 1-15, 1983.

16. Hashimoto, A., Onodera, T., Ikeda, H., and Kitani, H. Isolation and characterisation of fetal bovine brain cells in primary culture. Res Vet Sci 69, 39, 2000.

17. Jun, F., and Zhang, Z.X. Cryopreservation of embryonic cerebral tissue of rat. Cryobiology 29, 267, 1992.

18. Silani, V., Pizzuti, A., Strada, O., Falini, A., Buscaglia, M., and Scarlato, G. Human neuronal cell viability demonstrated in culture after cryopreservation. Brain Res 473, 169, 1988.

19. Fansa, H., Lassner, F., Kook, P.H., Keilhoff, G., and Schneider, W. Cryopreservation of peripheral nerve grafts. Muscle Nerve 23, 1227, 2000.

20. Phillips, J.B., and Brown, R. Micro-structured materials and mechanical cues in 3D collagen gels. Methods Mol Biol 695, 183, 2011.

21. Phillips, J.B. Monitoring neuron and astrocyte interactions with a 3D cell culture system. Methods Mol Biol 1162, 113, 2014.

22. East, E., Golding, J.P., and Phillips, J.B. A versatile $3 \mathrm{D}$ culture model facilitates monitoring of astrocytes undergoing reactive gliosis. J Tissue Eng Regen Med 3, 634, 2009.

23. Wright, K.E., Liniker, E., Loizidou, M., Moore, C., MacRobert, A.J., and Phillips, J.B. Peripheral neural cell sensitivity to mTHPC-mediated photodynamic therapy in a 3D in vitro model. Br J Cancer 101, 658, 2009.

24. Elder, E., Chen, Z., Ensley, A., Nerem, R., Brockbank, K., and Song, Y. Enhanced tissue strength in cryopreserved, collagen-based blood vessel constructs. Transplant Proc 37, 4625, 2005.

25. Costa, P.F., Dias, A.F., Reis, R.L., and Gomes, M.E. Cryopreservation of cell/scaffold tissue-engineered constructs. Tissue Eng Part C Methods 18, 852, 2012.

26. Mazur, P. Cryobiology: the Freezing of biological systems. Science 168, 939, 1970.

27. Kivell, B.M., McDonald, F.J., and Miller, J.H. Method for serum-free culture of late fetal and early postnatal rat brainstem neurons. Brain Res Brain Res Protoc 6, 91, 2001. 
28. Kivell, B.M., McDonald, F.J., and Miller, J.H. Serum-free culture of rat post-natal and fetal brainstem neurons. Dev Brain Res 120, 199, 2000.

29. Sauer, H., and Brundin, P. Effects of cool storage on survival and function of intrastriatal ventral mesencephalic grafts. Restor Neurol Neurosci 2, 123, 1991.

30. Nikkhah, G., Eberhard, J., Olsson, M., and Bjorklund, A. Preservation of fetal ventral mesencephalic cells by cool storage - in-vitro viability and th-positive neuron survival after microtransplantation to the striatum. Brain Res 687, 22, 1995.

31. Thompson, D.M., and Buettner, H.M. Neurite outgrowth is directed by Schwann cell alignment in the absence of other guidance cues. Ann Biomed Eng 34, 161, 2006.

32. Seggio, A.M., Narayanaswamy, A., Roysam, B., and Thompson, D.M. Self-aligned Schwann cell monolayers demonstrate an inherent ability to direct neurite outgrowth. J Neural Eng 7, 046001, 2010.

33. Richardson, J.A., Rementer, C.W., Bruder, J.M., and Hoffman-Kim, D. Guidance of dorsal root ganglion neurites and Schwann cells by isolated Schwann cell topography on poly(dimethyl siloxane) conduits and films. J Neural Eng 8, 046015, 2011.

34. Bellamkonda, R.V. Peripheral nerve regeneration: an opinion on channels, scaffolds and anisotropy. Biomaterials 27 , 3515, 2006.

Address correspondence to:

James B. Phillips, BSc, PhD

Department of Biomaterials and Tissue Engineering

UCL Eastman Dental Institute

University College London

256 Gray's Inn Road

London WC1X 8LD

United Kingdom

E-mail: jb.phillips@ucl.ac.uk

Received: May 2, 2017

Accepted: July 18, 2017

Online Publication Date: October 6, 2017 